\title{
化学自组装合成 $\mathrm{Sn}$ 掺杂的纳米 $\mathrm{TiO}_{2}$ 介孔材料 及其光催化性能*
}

\author{
张美红 丁士文** 王振兴 张玉卓
}

(河北大学化学与环境科学学院, 保定 071002 )

\begin{abstract}
摘要以 $\mathrm{TiCl}_{4} 、 \mathrm{SnCl}_{4}$ 为原料, 尿素为沉淀剂, 纳米级碳黑为模板, 可溶性淀粉为阻聚剂, 采用 微波加热, 均相沉淀法合成出了一系列 $\mathrm{Sn}$ 掺杂纳米 $\mathrm{TiO}_{2}$ 介孔材料. XRD 分析证明反应前驱体为 非晶态, $400^{\circ} \mathrm{C}$ 以上转变为锐钛矿结构. TEM 形貌观察, 粒子基本为球形, 平均粒径 $20 \mathrm{~nm}$. EDS 分 析证明产品中 $\mathrm{Ti}: \mathrm{Sn}$ 的分析测定值与实际的投料值基本一致, 并且掺杂均匀性好. 光吸收及光 催化实验发现 $\mathrm{Sn}$ 含量(摩尔浓度)为 $10 \%$ 时, 光催化效果最好, 在日光照射 $70 \mathrm{~min}$ 后, 此 $\mathrm{Sn}$ 掺杂 的 $\mathrm{TiO}_{2}$ 对藏蓝染料溶液降解率可达到 $100 \%$, 其光催化反应符合一级动力学方程.
\end{abstract}

\section{关键词 $\mathrm{Sn}$ 掺杂 $\mathrm{TiO}_{2}$ 微波合成 模板控制 均相沉淀 介孔材料 光催化}

自 1977 年Frank和Bard 首次报道了悬浮的 $\mathrm{TiO}_{2}$ 粉末光催化降解含 $\mathrm{CN}^{-}$溶液之后, 利用纳米半导体材 料光催化降解有害污染物已成为比较热门的研究课 题之一. 二氧化钛是一种重要的半导体光催化材料, 它具有光催化降解有机物活性高、化学性质稳定、耐 化学和光化学腐蚀以及无毒等特性, 因而在污水处 理及空气净化等方面有着重大的潜在应用价值 ${ }^{[1-4]}$. 然而二氧化钛是宽禁带材料, 仅能吸收太阳光谱的 紫外光部分, 通常需要用紫外光源来激发, 太阳能利 用效率低，这限制了其实际的应用. 为了提高二氧化 钛的光谱响应和太阳光的利用率, 人们提出了染料 敏化 ${ }^{[1]}$, 贵金属掺杂 ${ }^{[5]}$ 以及利用Sol-Gel技术进行半导
体-半导体掺杂自等方法对 $\mathrm{TiO}_{2}$ 进行改性的报道. 但 上述方法又均存在化学性质不稳定、成本高等弊端.

目前人们在许多化学领域运用微波技术进行了 卓有成效的工作, 自从 1888 年牛津大学的Baghurst 等在Nature杂志上首次报道用微波法进行了无机化 合物和超导材料的合成后，用微波技术合成沸石分 子管 ${ }^{[7]}$ 、无机材料 ${ }^{[8]}$ 等也陆续报道. 微波作为一种能 源, 正以比人们预料要快得多的速度步入化工、新材 料及其它高科技领域. 本文工作利用普通无机盐为 原料, 以碳黑为模板, 可溶性淀粉为阻聚剂, 利用微 波加热快速、均匀的特点, 采用均相沉淀法, 合成出 了粒度均匀可控的 $\mathrm{Sn}$ 掺杂的 $\mathrm{TiO}_{2}$ 介孔材料, 并对其 
光催化性能进行了研究.

\section{1 实验}

采用的原料 $\mathrm{TiCl}_{4}, \mathrm{SnCl}_{4}$ ，尿素，碳黑，淀粉等均 为国产分析纯试剂; 藏蓝染料为市售产品; 全部实验 用水为二次蒸馏水. 主要仪器有: Y-2000 型 X 射线衍 射仪, JEM-1000SX 透射电子显微镜, UV-1200 紫外 可见分光光度计, NORAN 能谱仪, KYKY-1000B 扫描 电子显微镜, Whirlpool 微波炉等.

纳米复合介孔材料的制备如下: 在 $500 \mathrm{~mL}$ 烧杯 中按照一定的化学计量比配置 $\mathrm{TiCl}_{4}$ 与 $\mathrm{SnCl}_{4}$ 的混合 溶液; 称取适量尿素溶于水, 加入盛有 $\mathrm{TiCl}_{4}$ 与 $\mathrm{SnCl}_{4}$ 溶液的烧杯中, 搅拌均匀, 溶液保持透明. 在上述溶 液中加入适量的碳黑作为模板剂, 可溶性淀粉作为 阻聚剂, 放入微波炉中加热至微沸, $3 \mathrm{~min}$ 后出现大 量白色沉淀, 保持微沸数分钟, 反应完毕, 冷却至室 温, 抽滤, 洗涤, 将滤饼在 $100^{\circ} \mathrm{C}$ 干燥, 得到反应前驱 体, 将前驱体在 $400^{\circ} \mathrm{C}$ 灼烧 $1 \mathrm{~h}$ 得到一系列 $\mathrm{Sn}$ 掺杂的 纳米 $\mathrm{TiO}_{2}$ 粉体, 分别编号为从 1 至 10 (见表 1). 实验 中对反应浓度、物料配比、反应时间等各种因素对样 品产量、粒度的影响作了详尽研究.

\section{表 1 样品}

\begin{tabular}{ccccccccccc}
\hline 样品编号 & 1 & 2 & 3 & 4 & 5 & 6 & 7 & 8 & 9 & 10 \\
\hline Sn 摩尔浓度 $/ \%$ & 0 & 2 & 4 & 6 & 8 & 10 & 12 & 14 & 16 & 18 \\
\hline
\end{tabular}

光吸收性能实验如下: 取适量系列 $\mathrm{Sn}$ 掺杂的纳 米 $\mathrm{TiO}_{2}$ 粉末均匀分散在水中, 超声波强力分散均匀 后, 用 UV-1200 紫外-可见分光光度计测定样品的吸 光度, 并与 Degussa-P25(德国 Degussa Corporation 生 产)和自制氧化钛进行了对比.

光催化降解效果测量在 UV-1200 紫外-可见分光 光度计上进行, 测量波长为 $200 \sim 800 \mathrm{~nm}$. 样品的制 备过程为: 配制 $50 \mathrm{mg} / \mathrm{L}$ 的藏蓝染料溶液, 取 $150 \mathrm{~mL}$ 置于 $250 \mathrm{~mL}$ 的雉形瓶中, 分别加入(从 1 10)75 mg 的纳米粉末, 磁力摚拌, 在太阳光照射下进行反应, 隔一定时间取样测试. 降解效果以降解率 $D(\%)$ 表示:

$$
D=\left[\left(A_{0}-A\right) / A_{0}\right] \times 100 \% .
$$

其中 $A_{0}$ 为染料溶液最大吸收峰的初始吸光度; $A$ 为染料溶液最大吸收峰的最终吸光度. 同时与 $\mathrm{P}_{25}$ 和
自制氧化钛进行了对比.

\section{2 结果与讨论}

\section{1 合成过程的影响因素}

\subsection{1 微波加热的优点}

尿素作为均相沉淀剂的反应机理为:

$$
\begin{gathered}
\mathrm{CO}\left(\mathrm{NH}_{2}\right)_{2}+3 \mathrm{H}_{2} \mathrm{O}=\mathrm{CO}_{2}+2 \mathrm{NH}_{4} \mathrm{OH} \\
\mathrm{Ti}^{4+}+\mathrm{Sn}^{4+}+8 \mathrm{OH}^{-}=\left[\mathrm{Ti}(\mathrm{OH})_{4}+\mathrm{Sn}(\mathrm{OH})_{4}\right] \downarrow
\end{gathered}
$$

尿素分子被加热到一定温度后(一般大于 $70^{\circ} \mathrm{C}$ ), 分解 出构晶离子 $\left(\mathrm{OH}^{-}\right)$, 与溶液中存在的 $\mathrm{Ti}^{4+}$ 和 $\mathrm{Sn}^{4+}$ 反应 生成氢氧化物沉淀, 后者脱水及热处理后得到纳米 复合粉体. 在纳米粒子制备过程中, 成核和生长是两 个关键步骤，产物粒子的大小取决于成核速度与晶 核生长速度的相对大小. 欲形成单分散的粒子, 要求 所有的核必须几乎同时形成，而且在接下去的生长 中没有进一步的成核或离子团聚。与常规加热不同, 微波加热不存在温度梯度, 快速而均匀, 可使沉淀相 瞬间萌发成核, 从而可获取均匀的纳米粒子.

\subsection{2 反应时间的影响}

在其它反应条件相同的条件下，改变反应时间 发现，反应时间过短，反应不充分，收率低. 随着反 应时间的延长, 收率明显提高, 但反应时间过长, 溶 液中的小晶粒溶解而大晶粒继续长大, 势必导致颗 粒尺寸变大. 实验证明当反应时间为 $20 \mathrm{~min}$ 时, 粒 子尺寸为 $20 \mathrm{~nm}$, 产率可达 $98 \%$ 以上，见图 1 .

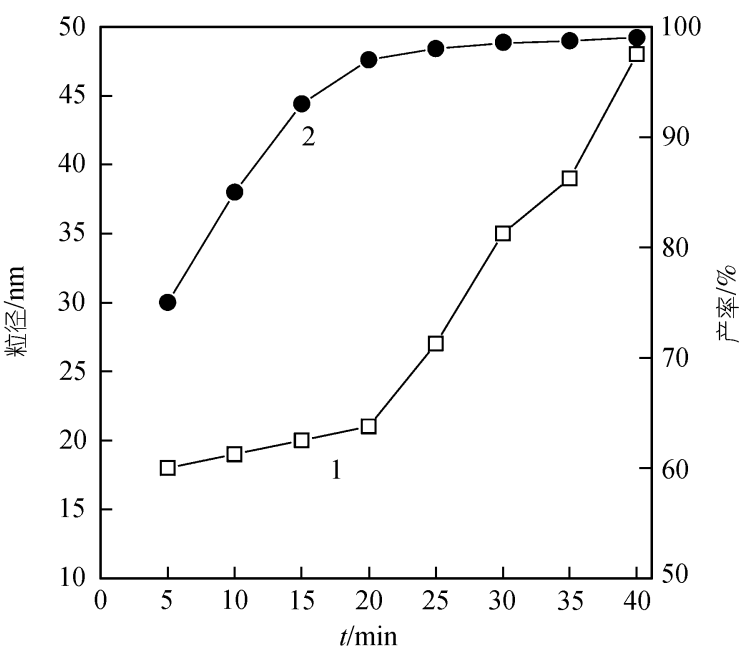

图 1 反应时间对样品粒径(1)和产率(2)的影响 


\subsection{3 反应物配比的影响}

产物粒子尺寸大小与反应物配比有很大关系. 产物粒子尺寸先是随着沉淀剂的加入量的增多而减 小, 而后又随着加入量的增加而增大. 实验证明 $\mathrm{Ti}^{4+}$ 和 $\mathrm{Sn}^{4+}$ 与尿素的最佳摩尔配比为 $1: 2$, 见图 2 .

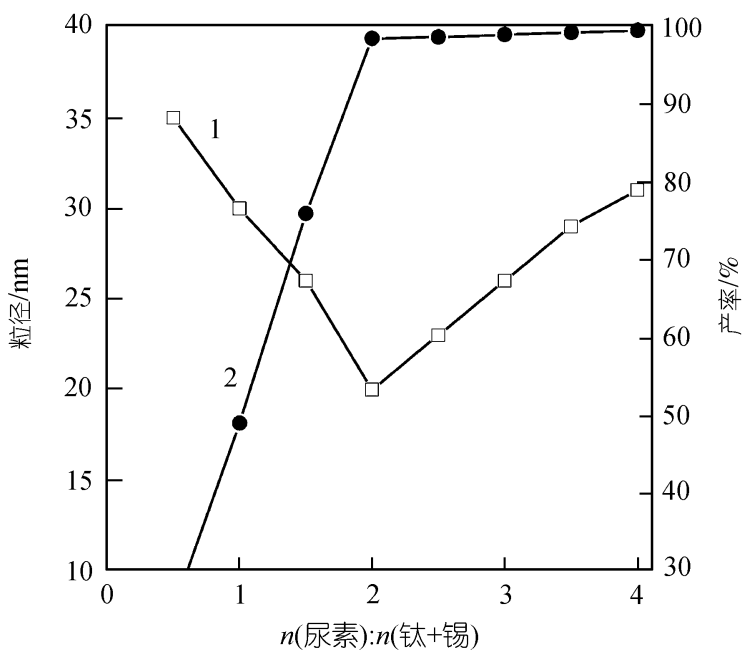

图 2 反应物料配比对样品粒径(1)和产率(2)的影响

\subsection{4 模板和阻聚剂的作用}

纳米微粒由于它们具有大的比表面积，常常团 聚构成二次粒子. 细小粒子的团聚可能发生在合成 阶段、干燥过程及后来的处理中, 因此主要的是粒子 制备及后处理的每一步都使粒子处于稳定状态而不 团聚。本实验利用可溶性淀粉等分散剂的大分子链 的空间位阻效应，高分子网络的阻隔作用，并配合固 体碳黑模板的防团聚的作用，使产品粒径大幅度减 小; 在 $400^{\circ} \mathrm{C}$ 烧碳后，粒子间会留下大量空穴，从而 得到粒径分布均匀的纳米介孔材料(见图 3).

\subsection{TEM 形貌分析}

TEM 形貌分析证明粒子基本为球形，平均粒径 约为 $20 \mathrm{~nm}$. 且粒度分布很窄. 通过控制一定的模板 剂和阻聚剂用量, 可以由纳米粒子自组装成外貌为 条形，尺寸为 100 200 nm 的介孔材料. 这些介孔材 料的优点是：(1) 在保存过程中, 可以进一步防止纳 米粒子之间的硬团聚; (2) 作为光催化材料, 高分散 纳米粒子在制备和回收过程中的过滤问题是一个大
的难题, 而介孔材料巧妙的解决了这个问题, 它即保 留了纳米材料的高催化活性，又容易通过过滤而回 收.
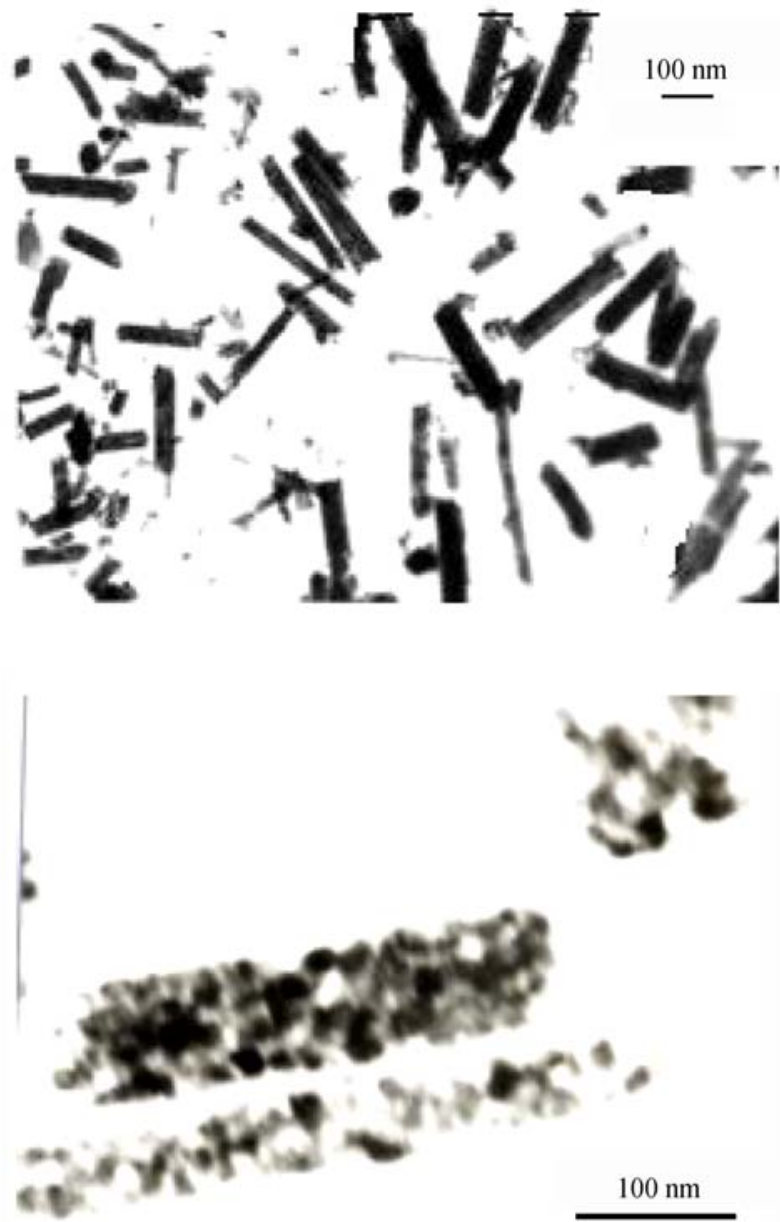

图 3 样品的 TEM 照片

\section{3 样品的 XRD 分析}

对不同 $\mathrm{Sn}, \mathrm{Ti}$ 比的系列样品分别进行 XRD 分析, 发现前驱体均为非晶态(图 4(2)), 在 $400^{\circ} \mathrm{C}$ 转变为结 晶的锐钛矿结构(图 4(1)), 从图中并未发现 $\mathrm{SnO}_{2}$ 的衍 射峰, 说明掺入的 $\mathrm{Sn}^{4+}$ 已经进入了 $\mathrm{TiO}_{2}$ 的晶格.

\section{4 样品的 EDS 分析}

将制得的一系列不同 $\mathrm{Sn}$ 与 $\mathrm{Ti}$ 配比的复合材料进 行 $\operatorname{EDS}(\mathrm{X}$-射线能量散射谱)分析，结果表明产品中 $\mathrm{Sn}$ ： $\mathrm{Ti}$ 的分析测定值与实际的投料值基本一致，图 5 
为含锡 $10 \%$ 样品的 EDS 图谱, 图谱分析结果表明: $\mathrm{Sn}$ 含量(摩尔浓度)为 $9.95 \%, \mathrm{Ti}$ 为 $90.05 \%$, 而实际投 料值为 $\mathrm{Sn}: 10 \%$, Ti: $90 \%$ ，两者基本一致，其微小的 差别可能是样品称量中的误差所至. 证明了该合成 方法是科学、可靠的.

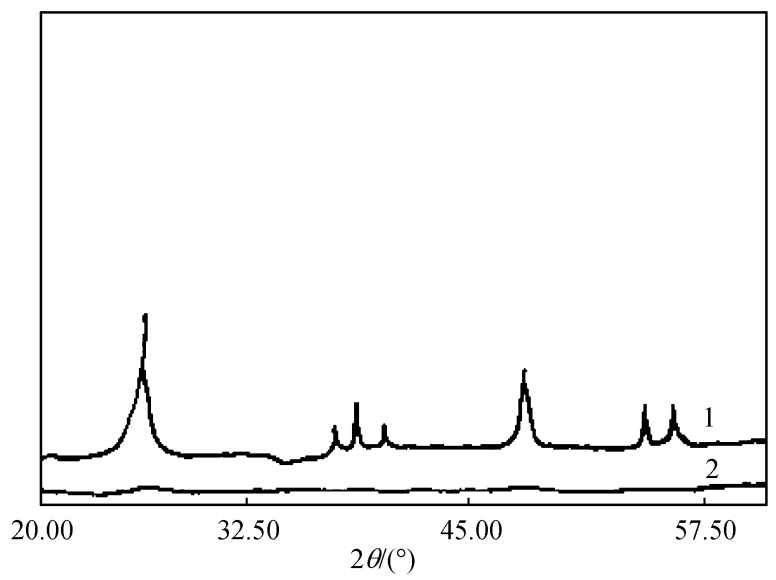

图 4 样品的 XRD 图谱

1 , 在 $400^{\circ} \mathrm{C}$ 灼烧; 2 , 未灼烧

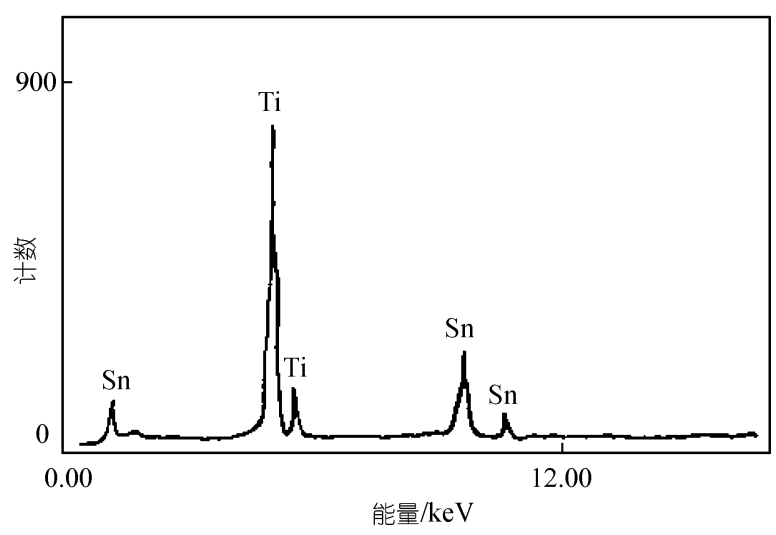

图 5 样品的 EDS 图谱

同时通过面分布图可以看出 $\mathrm{Ti}$ 和 $\mathrm{Sn}$ 的分布是均 匀的(图 6), 表明在样品的制备过程中 $\mathrm{Ti}$ 和 $\mathrm{Sn}$ 已经形 成了均匀的体系. 样品具有较高的均匀性.

\section{5 光吸收性能及光催化性能}

将制得的一系列产品进行光吸收实验，发现样 品的最大光吸收值 $\left(A_{\max }\right)$ 随着 $\mathrm{Sn}$ 掺入量的增大先增 大而后又逐渐减小，同时对藏蓝染料进行光催化实
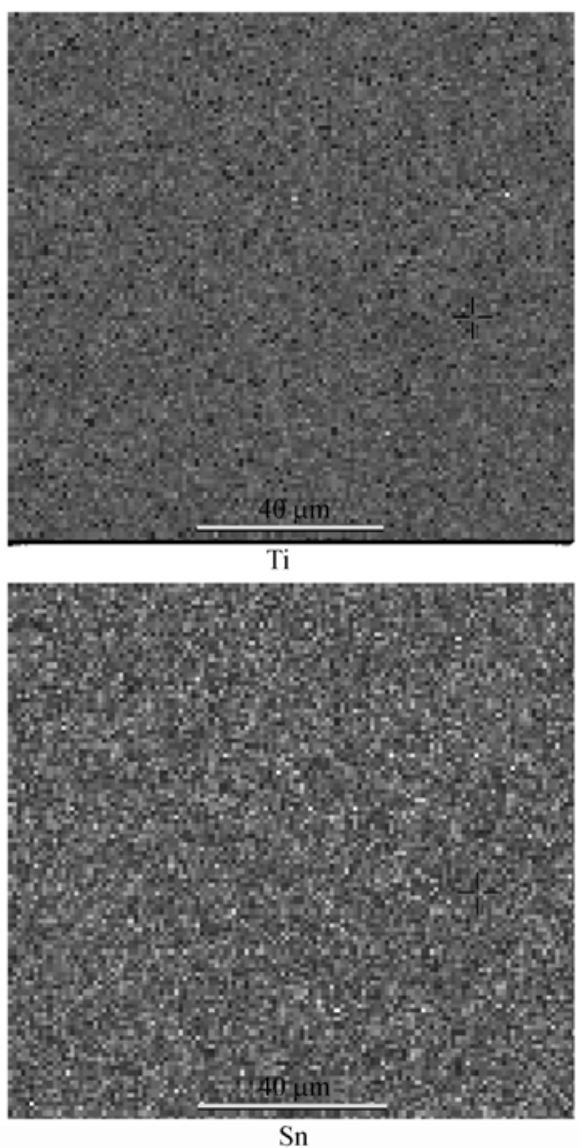

图 6 样品中 $\mathrm{Ti}, \mathrm{Sn}$ 的面分布图

验(太阳光照射 $70 \mathrm{~min}$ )发现光催化降解率 $(D \%)$ 具有 相同的规律，结果见表 2. 通过表中的数据可以看出: 样品 6 的 $A_{\max }$ 值最大, 对藏蓝染料的光降解率可接近 $100 \%$, 掺杂样品的光吸收性能及对藏蓝染料的光催 化性能明显好于 $\mathrm{P}_{25}$ 样品及其及自制的纯 $\mathrm{TiO}_{2}$, 可作 为光催化剂应用于染料溶液的光催化降解实验中. 这主要是因为, 掺杂可以形成掺杂能级, 使能量较小 的光子能激发掺杂能级上捕获的电子和空穴，从而 提高光子的利用率. 另外, 掺杂可以导致载流子的扩 散长度增大, 从而延长了电子和空穴的寿命，抑制了 $\mathrm{e}^{-} / \mathrm{h}^{+}$复合，最终导致复合材料的太阳能利用率的提 高, 光催化效果显著 ${ }^{[1]}$.

图 7 是藏蓝染料溶液在不同时间太阳光照射降 解后的紫外-可见光谱图(用样品 6 做光催化材料), 由 谱图可见, 随着光催化反应时间的延长, 溶液的紫外- 
表 2 样品的最大光吸收值及对藏蓝染料的光降解率

\begin{tabular}{|c|c|c|c|c|c|c|c|c|c|c|c|}
\hline 样品 & $\mathrm{P}_{25}$ & 1 & 2 & 3 & 4 & 5 & 6 & 7 & 8 & 9 & 10 \\
\hline$A_{\max }$ & 0.828 & 0.621 & 0.803 & 1.065 & 1.326 & 1.467 & 1.684 & 1.506 & 1.286 & 1.201 & 1.002 \\
\hline$D / \%$ & 65.2 & 42.6 & 64.5 & 78.2 & 92.1 & 95.1 & 100 & 97.3 & 90.6 & 87.7 & 72.3 \\
\hline
\end{tabular}

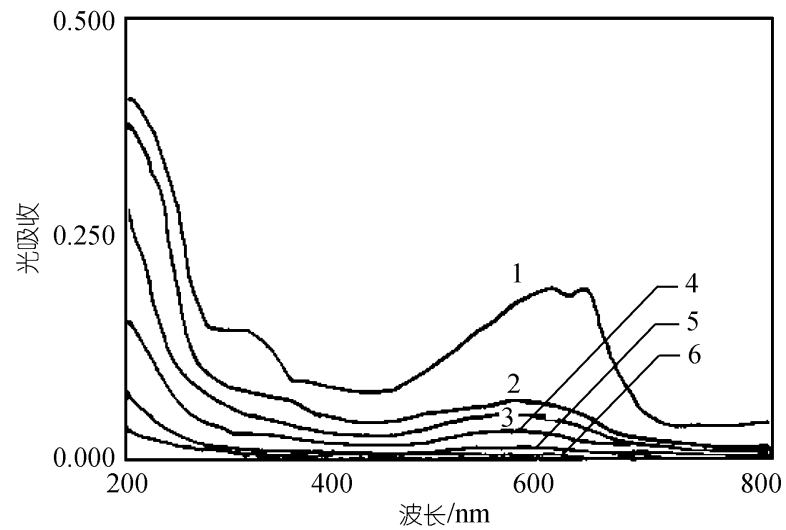

图 7 光催化 藏蓝染料的 $A-\lambda$ 扫描曲线

1, 未光照; 2, $30 \mathrm{~min} ; 3,40 \mathrm{~min} ; 4,50 \mathrm{~min} ; 5,60 \mathrm{~min} ; 6,70 \mathrm{~min}$

可见光吸收逐渐减小. 直到 $70 \mathrm{~min}$ 后溶液在紫外-可 见光范围内已无吸收，计算脱色效率为 $100 \%$. 用重 铬酸钾法测定其相应的 $\mathrm{COD}_{\mathrm{Cr}}$ 去除率亦接近 $100 \%$. 说明采用 $\mathrm{Sn}$ 掺杂的纳米 $\mathrm{TiO}_{2}$ 光降解染料溶液不仅能 迅速破坏染料中的发色基团，而且能有效地破坏染 料分子中的芳香基团. 其基本原理是，纳米微粒由于 尺寸小, 表面所占的体积百分数大, 表面的键态和电 子态与颗粒内部不同，表面原子配位不全等导致表 面的活性位置增加，且随着粒径的减小，表面光滑程 度变差, 形成了凸凹不平的原子台阶, 这就增加了纳 米材料的吸附性和化学反应的接触面. 另外, Sn 掺杂 的纳米 $\mathrm{TiO}_{2}$ 由于量子尺寸效应使其导带和价带能级 变成分裂能级. 因此当 $\mathrm{Sn}$ 掺杂的纳米 $\mathrm{TiO}_{2}$ 受到太阳 能的辐射后，处于价带的电子就被激发到导带，价带 便生成空穴 $\left(\mathrm{h}^{+}\right)$, 空穴 $\mathrm{h}^{+}$本身是强氧化剂将吸附在 $\mathrm{Sn}$ 掺杂的纳米 $\mathrm{TiO}_{2}$ 多孔材料表面的 $\mathrm{OH}^{-}$和 $\mathrm{H}_{2} \mathrm{O}$ 分子氧 化生成 $\mathrm{OH}$ - 自由基, 缔合在纳米材料表面的 $\mathrm{OH} \cdot$ 为 强氧化剂, 可以氧化相邻的有机物, 而且可以扩散到 液相中氧化有机物, 把各种有机物通过一系列的氧 化过程, 最终氧化成 $\mathrm{CO}_{2}$, 从而完成对有机物的降 解.

\section{6 反应动力学}

以样品 6 处理藏蓝染料为例, 对 $\ln \left(C_{0} / C_{t}\right)$ 与光照 时间 $t$ 作图, 结果如图 8 所示. 发现 $\ln \left(C_{0} / C_{t}\right)$ - $t$ 之间基 本具有直线关系，表明复合光催化剂材料的光催化 反应仍为一级反应. 其速率方程可表示为: $\ln \left(C_{0} / C_{t}\right)=$ $K t$, 式中 $K$ 是表观反应速率常数, $C_{0}$ 和 $C_{t}$ 分别是染料 溶液的起始质量浓度和 $t$ 时的质量浓度. 动力学方程 及动力学常数为:

$$
\begin{aligned}
\ln \left(C_{0} / C_{t}\right)= & 0.01356 t+0.06075, K=0.01356, \\
R= & 0.99287, C_{0}=50.00 \mathrm{mg} / \mathrm{L}, \\
& \text { 催化剂加入量 } 0.70 \mathrm{~g} / \mathrm{L} .
\end{aligned}
$$

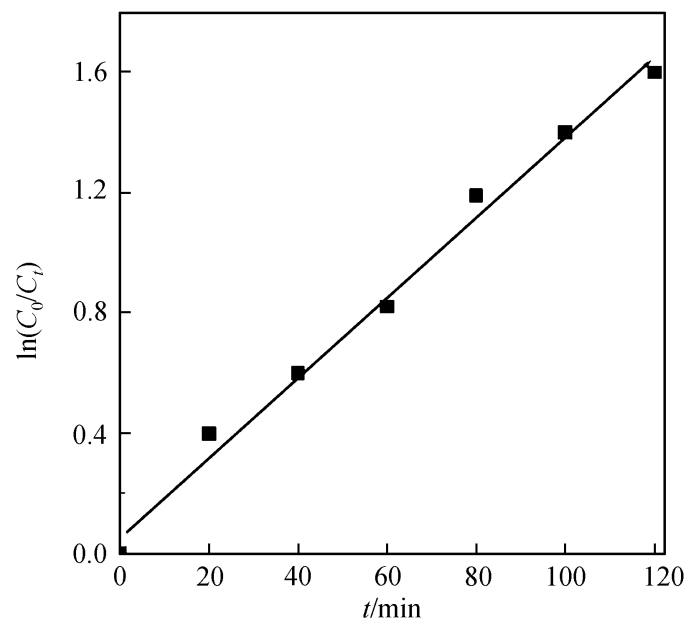

图 8 浓度 $\ln \left(C_{0} / C_{t}\right)$ 与光照时间的关系

\section{3 结论}

（1）以普通无机盐为原料，利用微波加热快速、 均匀的特点; 用碳黑作为模板和分散剂, 利用可溶性 淀粉大分子链的空间位阻效应，采用化学自组装制 备出了粒度分布均匀、对紫外-可见光均敏感的 Sn 掺 杂的纳米 $\mathrm{TiO}_{2}$ 介孔材料.

(2) 光吸收及光催化实验发现 $\mathrm{Sn}$ 与 $\mathrm{Ti}$ 摩尔比为 $0.10: 0.90$ 时其光吸收性能与光催化性能最好, 在太 
阳光下照射 $70 \mathrm{~min}$ 后藏蓝染料溶液的降解率可达到 $100 \%$, 复合材料的光催化反应仍然符合一级动力学 方程.

（3）该介孔催化剂不仅催化活性高，而且极容易 过滤回收使用.

\section{参考文 献}

1 张立德, 牟季美. 纳米材料和纳米结构. 北京: 科学出版社, 2001. 59 88

2 Zhan Haoqiang, Chen Kongchang, He Tian. Photocatalytic Degradation of Acid Azo Dyes in Aqueous $\mathrm{TiO}_{2}$ Suspension. II. The Effect of pH Values. Dyes and Pigments, 1998, 37: 241 247[DOI]

3 冯良荣, 谢卫国, 吕绍洁, 等. 纳米 $\mathrm{TiO}_{2}$ 催化剂微晶结构对光
催化反应的影响. 中国科学, B 辑, 2001, 31(6): 536 541

4 贺北平, 王占生, 张锡辉. 半导体光催化有机物的研究现状及 发展趋势. 环境科学, 1994, 15(3): 80 83

5 Falconer J L, Magrini B, Kimberley A. Photocatalytic and thermal catalytic oxidation of acetaldehyde on $\mathrm{Pt} / \mathrm{TiO}_{2}$. Journal of Catalysis, 1998, 179(2): 171 178[DOI]

6 Yuan Zhihao, Jia Junhui, Zhang Lide. Influence of co-doping of $\mathrm{Zn}(\mathrm{II})+\mathrm{Fe}(\mathrm{III})$ on the photocatalytic activity of $\mathrm{TiO}_{2}$ for phenol degradation. Materials Chemistry and Physics, 2002, 73(4): 323 326[DOI]

7 庞广生, 崔得良, 徐如人. 微波固相法合成层状磷钩酸钾化合 物. 化学学报, 1996, 54(6): 575 580

8 冯守华, 庞广生, 徐如人. 微波诱导合成固体快离子导电材料. 高等学校化学学报, 1996, 17(10): 1495 1499 